$$
\text { DOE/ER/54/22--73 }
$$

\title{
ROBUSTNESS OF RADIATIVE MANTLE PLASMA POWER EXHAUST SOLUTIONS FOR ITER
}

\author{
J. Mandrekas, W. M. Stacey, F.A. Kelly \\ Fusion Research Center \\ Georgia Institute of Technology \\ Atlanta, GA 30332 USA \\ September, 1996
}

\begin{abstract}
The robustness of impurity-seeded radiative mantle solutions for ITER to uncertainties in several physics and operating parameters is examined. Our results indicate that $\sim 50-90 \%$ of the input power can be radiated from inside the separatrix with $\mathrm{Ne}, \mathrm{Ar}$ and $\mathrm{Kr}$ injection, without significant detriment to the core power balance or collapse of the edge temperature profile, for a wide range of conditions on the impurity pinch velocity, edge temperature pedestal, and plasma density.
\end{abstract}

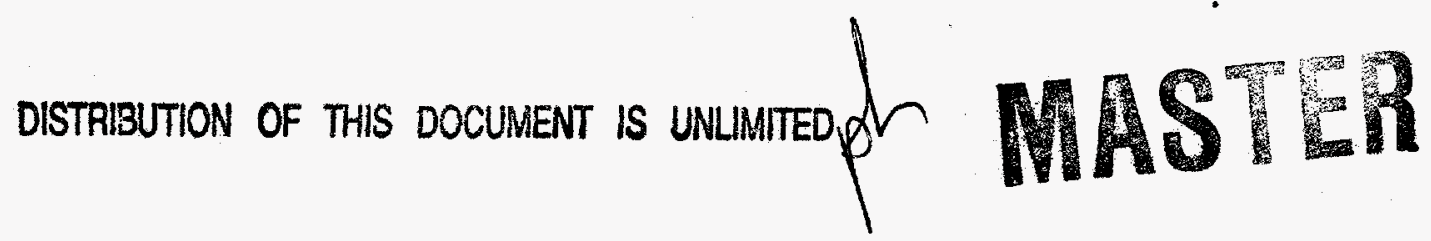




\section{INTRODUCTION}

Radiative power exhaust from within the separatrix and, in particular, radiation from the plasma mantle enhanced by the injection of impurities, is a significant component of any power exhaust solution that relies on atomic processes to disperse the heating power in the International Thermonuclear Experimental Reactor (ITER) [1]. Our previous work [2-4] established the feasibility of the impurity-seeded radiative mantle for ITER, showing that a substantial amount of the plasma power exhaust in ITER can be radiated from within the separatrix with the injection of different impurity species, without significant detriment to the core power balance.

These findings are supported by recent experimental results from TEXTOR [5-7], ASDEX-Upgrade [8] and DIII-D [9]. The TEXTOR experiments demonstrated the feasibility of obtaining a stable radiating layer with a ratio of radiated to input power, $\gamma$, exceeding $90 \%$ by injecting silicon and/or neon, without loss of confinement (confinement improvement was actually observed at high densities) or accumulation of the radiating impurity in the center. The results from the ASDEX-Upgrade confirmed the compatibility of high core confinement and enhanced main chamber radiation from $\mathrm{Ne}$ and $\mathrm{Ar}$ for operation above but close to the $\mathrm{H}$ to $\mathrm{L}$ transition threshold.

The main goal of the present work is to establish the robustness of the radiative mantle solution to inherent uncertainties in several important physical and operational parameters in the design of a next generation reactor such as ITER. In particular, we study the sensitivity of such solutions to thermal and impurity transport assumptions, to different values of the edge temperature pedestal, and to alternative ITER operating points including operation at the Greenwald density limit.

The paper is organized as follows: A summary of the computational models and transport assumptions is given in Section 2. The results of analyses are discussed in Section 3, and conclusions follow in Section 4.

\section{MODELS AND ASSUMPTIONS}

\subsection{Equations}

Our simulations have been performed using GTWHIST, the Georgia Tech version of the $11 / 2$ D plasma transport code WHIST [10]. The code solves the standard set of the flux surface averaged particle and energy balance and poloidal flux diffusion equations, on a 2-D flux surface geometry calculated by a fixed boundary MHD code. In addition, the code includes routines for the radial transport of all the charge states of several impurity species and a "2-point" SOL/divertor model with impurity radiation. The atomic rate coefficients needed in the calculation of the impurity radiation and transport are computed using the ADPAK package. A detailed description of the physical models in GTWHIST can be found in Refs. $3 \& 4$.

\subsection{Transport Models}

We have adopted the transport recommendations of the ITER Joint Central Team (JCT) [11]. Since a theory-based model has not yet been recommended by the ITER 


\section{DISCLAIMER}

This report was prepared as an account of work sponsored by an agency of the United States Government. Neither the United States Government nor any agency thereof, nor any of their employees, makes any warranty, express or implied, or assumes any legal liability or responsibility for the accuracy, completeness, or usefulness of any information, apparatus, product, or process disclosed, or represents that its use would not infringe privately owned rights. Reference herein to any specific commercial product, process, or service by trade name, trademark, manufacturer, or otherwise does not necessarily constitute or imply its endorsement, recommendation, or favoring by the United States Government or any agency thereof. The views and opinions of authors expressed herein do not necessarily state or reflect those of the United States Government or.any agency thereof. 


\section{DISCLAIMER}

Portions of this document may be illegible electronic image products. Images are produced from the best available original document. 
Confinement Modeling and Database' Expert group, we are using a fixed-shape empirical local transport model. Our local transport coefficients have the form:

$$
\begin{gathered}
\chi_{c}=C \frac{a^{2}}{\tau_{\text {off }}} F(\rho) \\
\chi_{i}=2 \chi_{e} \\
D=0.5 \chi_{i}
\end{gathered}
$$

where $\tau_{\triangle f}=\min \left[\tau_{M A}, \tau_{E}^{I T E R H 93 P}\right], \tau_{N A}$ is the neo-Alcator (ohmic) confinement time, $\tau_{E}^{\text {ITERH } 93 P}$ is the ITER ELMy H-Mode thermal confinement scaling [13],

$$
\tau_{E}^{\Pi E R H 93 P}=0.036 I_{P}^{1.06} B_{T}^{0.32} M^{0.41} n_{19}^{0.17} R^{1.90} a^{-0.11} K^{0.66} P_{L}^{-0.67}
$$

and the coefficient $C$ is adjusted at every time step so that the total global energy confinement time is $0.85 \times \tau_{E}^{\text {TERH } 93 P}$. It should be noted here that in our calculations the radiation corrected heating power term in Eq. 4, $P_{L}$, is defined as $P_{L}=P_{\text {in }}-P_{\text {rad }}^{\text {core }}$ where $P_{\text {in }}$ is the total heating power and $P_{\text {rad }}^{\text {care }}$ is the total radiation power from inside the $q=2$ surface. This is done to ensure that the confinement time will remain finite in the enhanced radiation cases, where a large fraction of the input power is radiated outside the $q=2$ surface. No anomalous particle pinch is assumed, which results in flat density profiles, in accordance with the JCT guidelines. The shape of the transport coefficients is determined by the profile factor $F(\rho)$ which has the form,

$$
F(\rho)=\left(1+\lambda \rho^{2}\right) \exp \left(a \rho^{2}+b \rho^{4}\right)
$$

where $\lambda, a$ and $b$ are adjustable coefficients. This profile factor is very versatile and, with a proper choice of the coefficients $\lambda, a$ and $b$, it can give us transport coefficients with shapes that match many of the experimentally determined coefficients in various confinement regimes.

In our calculations, the coefficients $\lambda, a$ and $b$ are computed so that the resulting profiles have prescribed values for the edge-to-center ratio, $\chi_{e}(1) / \chi_{e}(0)$, location of the peak, $\rho_{.}$, and peak-to-center ratio, $\chi_{e}\left(\rho_{\bullet}\right) / \chi_{e}(0)$. A typical profile, similar to the one used in the present simulations, is shown in Fig. 1. The reduced level of transport near the plasma edge helps to raise the edge pressure gradient and the edge temperature pedestal.

Within the scrape-off-layer (SOL) region, constant transport coefficients, $D_{\perp}=\chi_{e \perp}=\chi_{i \perp}=0.1 \mathrm{~m}^{2} / \mathrm{s}$, have been used. This level of edge transport results in a SOL with a power width of about $1 \mathrm{~cm}$.

The thermal alphas are transported with the same coefficients as the main ions. The wall boundary condition for the thermal alphas is adjusted so that the helium levels satisfy the JCT guidelines of $\tau_{H e}^{*} / \tau_{E}=10$ where $\tau_{H e}^{*}=\left\langle n_{H e}\right\rangle V / \Gamma_{H e}, V$ is the plasma volume and $\Gamma_{H e}\left(\mathrm{~s}^{-1}\right)=3.571 \times 10^{17} P_{\text {fusion }}(\mathrm{MW})$. 


\subsection{Reference Ignited Operating Point and Comparison with PRETOR Profiles}

We first performed transport simulations in order to establish a reference operating point for the ITER EDA design parameters $\left(R_{0}=8.14 \mathrm{~m}, a=2.80 \mathrm{~m}, P_{\text {fusion }}=1.5 \mathrm{GW}, I_{p}\right.$ $=21 \mathrm{MA}$ ) and to compare our plasma profiles to those predicted by the JCT code PRETOR [12].

A $2 \%$ uniform Beryllium fraction is assumed. In addition, a sawtooth crash is triggered every 50 seconds, during which the energy and particle profiles and the current densities are redistributed inside the mixing radius according to Kadomtsev's reconnection model.

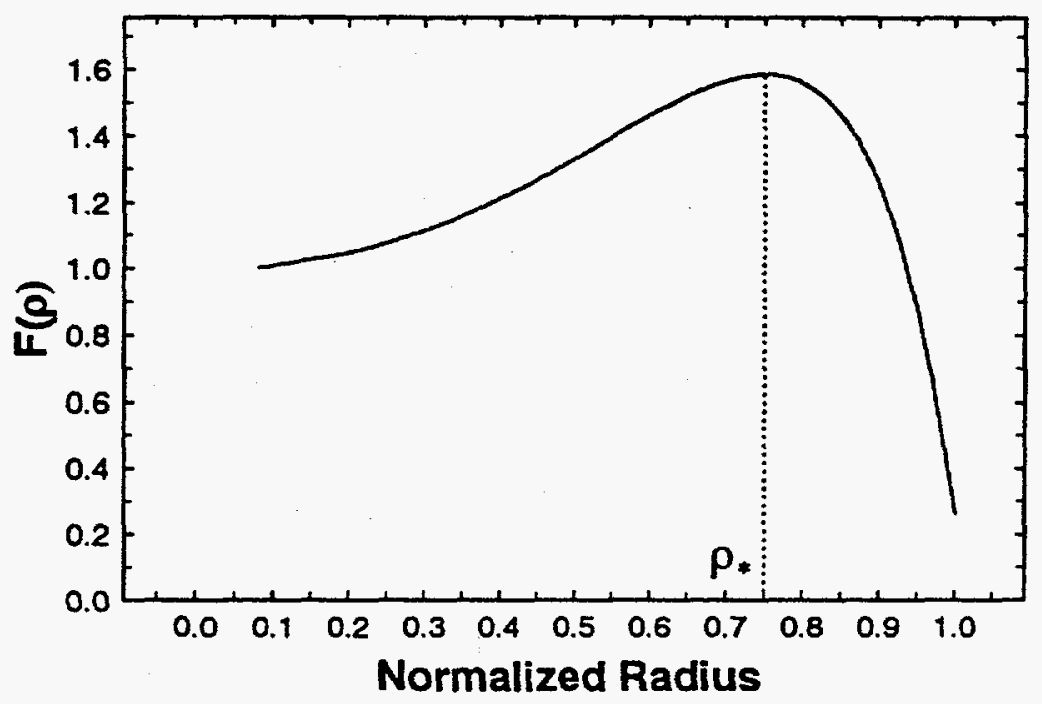

Fig. 1: Profile factor (Eq. 5) with $\lambda=-0.971, a=2.341$, and $b=-0.177$ corresponding to $\chi_{e}(1) / \chi_{e}(0)=0.25, \rho_{s}=0.75$ and $\chi_{e}\left(\rho_{c}\right) / \chi_{e}(0)=1.6$.

Our reference ignited operating point has the following global parameters: $P_{\text {fusion }}=$ $1500 \mathrm{MW}, P_{\text {rad }}=90 \mathrm{MW},\left\langle T_{e}\right\rangle=9.5 \mathrm{keV},\left\langle T_{i}\right\rangle=8.8 \mathrm{keV},\left\langle n_{e}\right\rangle=1.32 \times 10^{20} \mathrm{~m}^{-3}$. The electron temperature at the separatrix was about $340 \mathrm{eV}$, and the total power to the divertor plates was equal to $207 \mathrm{MW}$.

The temperature and density profiles for our reference case are shown in Figs. 2 4. For comparison, the JCT reference profiles, as predicted by the PRETOR code, are also included. It can be seen that the agreement in the electron temperatures and densities is very good, especially near the edge where the GTWHIST electron temperature profile and its gradient agree quite well with the PRETOR profile. The agreement between the ion temperature profiles (Fig. 3) is not as good near the center. This may reflect differences in the fusion reactivity models in the two codes. Notice also that in Figs. 2-4 the GTWHIST profiles extend beyond the last closed flux surface (LCFS) into the SOL.

We conclude from this comparison that the GTWHIST and PRETOR calculations are in sufficient agreement for the purpose of design analysis. 


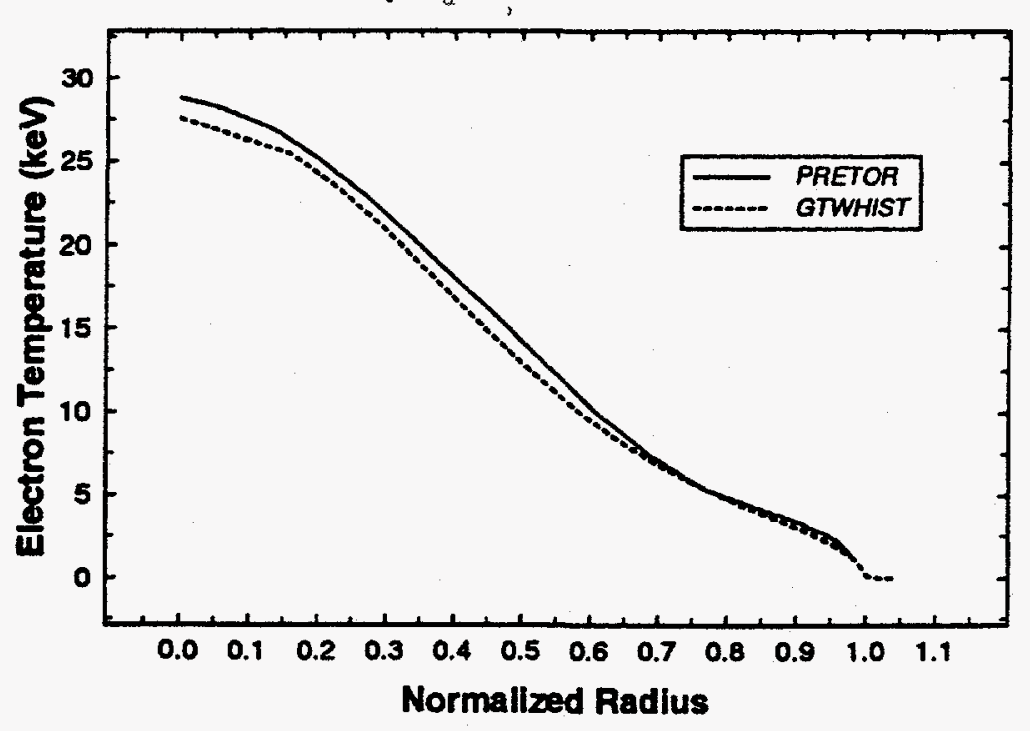

Fig. 2: Comparison of electron temperature reference profiles.

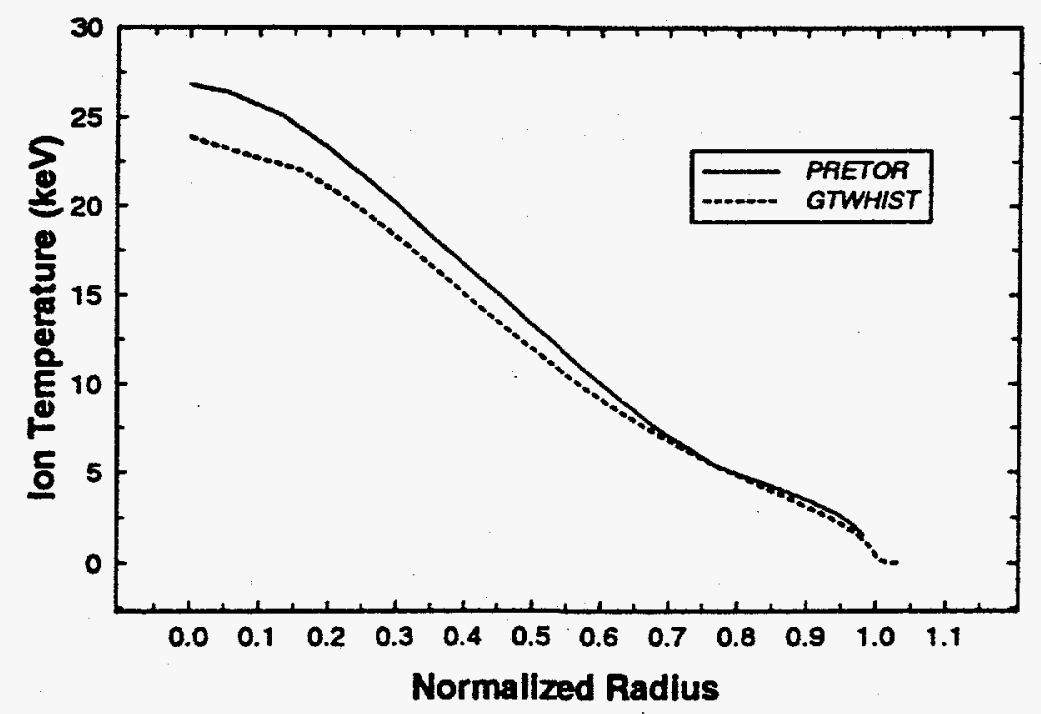

Fig. 3: Comparison of ion temperature reference profiles.

\section{INVESTIGATIONS OF IMPURITY-SEEDED RADIATIVE POWER EXHAUST IN ITER}

\subsection{Reference Transport Assumptions}

Having established an operating point consistent with the JCT asșumptions, we have performed radiative mantle simulations with injection of $\mathrm{Ne}, \mathrm{Ar}$ and $\mathrm{Kr}$ impurities. These simulations are similar to the ones reported in Ref. 3, but updated to be consistent with the most recent ITER design parameters, reference operating point assumptions and reference transport model. 


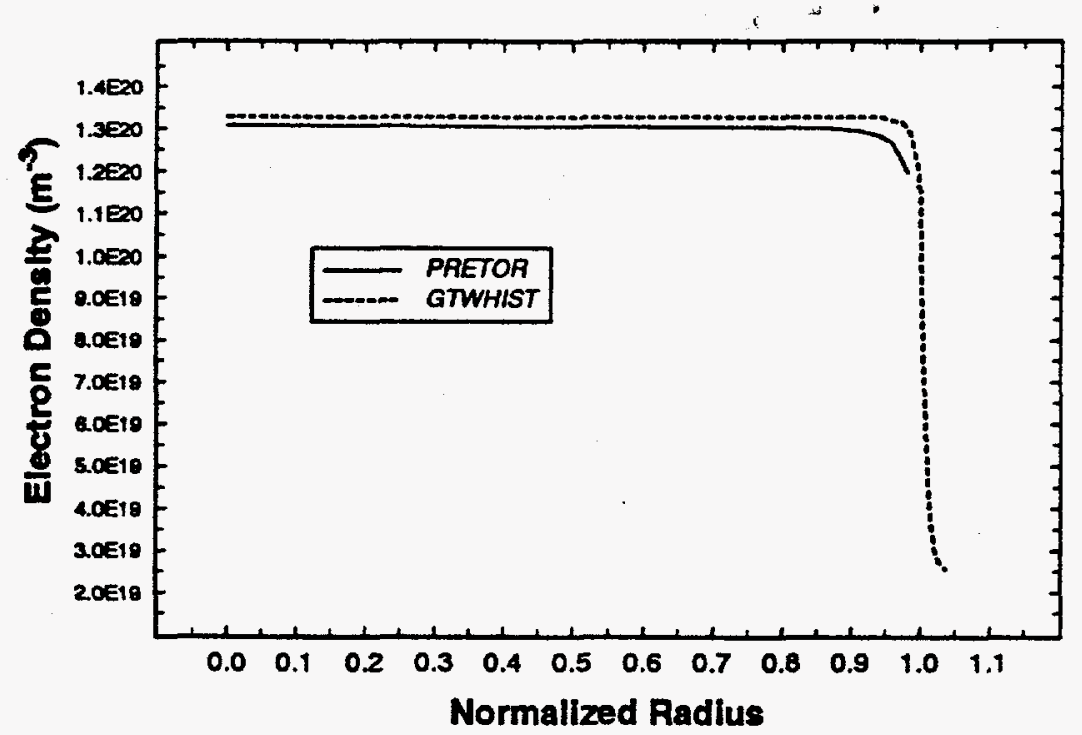

Fig. 4: Comparison of electron density reference profiles

The diffusion coefficient for all impurity charge states is assumed to be equal to the main ion particle diffusion coefficient (Eq. 3), and there is no impurity pinch. The effect of impurity seeding on the core power balance was compensated by auxiliary heating. The same core performance constraints that were used in Ref. 3 on the reduction of the fusion power $\left(\Delta P_{\alpha} / P_{\alpha} \leq 5 \%\right)$ and the plasma $Q(\geq 25)$ following impurity injection have been retained in these simulations as well.

The results from these simulations are shown in Figs 5-7, where the radiation fractions from different plasma regions (total, mantle ${ }^{1}$, core ${ }^{1}$, SOL/divertor) are shown versus the impurity concentration $f_{z}=\left\langle n_{z}\right\rangle /\left\langle n_{c}\right\rangle$ for Ne, Ar and Kr. In Fig. 8, various powers (total input power $P_{I N}=P_{\text {aux }}+P_{\text {alpha, }}$, total radiated power $P_{R A D T O T}$, radiated power from inside the separatrix $P_{R A D N}$, total bremsstrahlung power $P_{B R E M}$, radiated power from the SOL and divertor regions $P_{S O L}$, and total power to the divertor plates, $\left.P_{D N v}\right)$ are shown versus the impurity concentration $f_{z}$ for the case of Ar. From these figures it can be seen that up to $85-90 \%$ of the input power can be radiated, most of it originating from inside the separatrix as impurity line radiation from the edge or bremsstrahlung from the plasma core.

We note that our simple edge model probably underpredicts the amount of radiation from the SOL/divertor region. A more accurate treatment of the impurity transport in the SOL/divertor region may predict more radiation from this region.

The results for the maximum allowable impurity concentration which could be tolerated without violating our performance constraints or causing a collapse of the temperature profile at the edge, are summarized in Table 1.

\footnotetext{
${ }^{1}$ In our work we define the plasma mantle to be the region between the $q=2$ surface and the LCFS and the plasma core to be the region inside the $q=2$ surface.
} 


\begin{tabular}{|c|c|c|c|c|}
\hline & No Inj. & $\mathrm{Ne}$ & $\mathbf{A r}$ & $\mathbf{K} \mathbf{r}$ \\
\hline Total Radiated Power (MW) & 90 & 255 & 303 & 293 \\
\hline Radiated Fraction of Input Power, $\gamma$ & 0.29 & 0.73 & 0.85 & 0.90 \\
\hline Core Radiation (MW) & 71 & 131 & 152 & 120 \\
\hline Mantle Radiation (MW) & 4 & 54 & 108 & 165 \\
\hline SOLDivertor Radlation (MW) & 14 & 70 & 43 & 9 \\
\hline Power Across Separatrix (MW) & 218 & 158 & 86 & 35 \\
\hline Total Power to Divertors (MW) & 204 & 88 & 43 & 26 \\
\hline Impurity Fraction $f_{z}(\%)$ & $\mathbf{0}$ & 0.75 & 0.16 & 0.0132 \\
\hline Central Zoff & 1.48 & 2.14 & 1.95 & 1.61 \\
\hline Te@edge (eV) & 340 & 253 & 160 & 93 \\
\hline$Q=P_{\text {fuation }} / P_{\text {axx }}$ & $\infty$ & 26 & 25 & 57 \\
\hline
\end{tabular}

Table 1: Maximum radiated powers and related quantities for different impurity species at maximum impurity concentrations.

We observe that the radiation fractions obtained here, are higher than those calculated with our earlier transport and profile assumptions [3] (58\% for $\mathrm{Ne}, 65 \%$ for $\mathrm{Ar}$ and $73 \%$ for $\mathrm{Kr}$ ). This appears to be due to the reduced level of transport at the plasma edge predicted by the present transport model, which creates a "trap" for the injected impurities at the plasma edge allowing higher radiation fractions without violating any of our core performance constraints. In the present simulations, the maximum impurity concentration for $\mathrm{Ne}$ and $\mathrm{Kr}$ is limited only by the thermal stability of the plasma edge as evidenced by the collapse of the temperature profile at the edge. The maximum concentration for Ar is limited by the constraint on the plasma $Q$.

It should be noted here that our simulations, and hence the results in Table 1, do not account for any confinement degradation due to a possible H-to-L transition caused by the reduction of the power crossing the separatrix. To address this issue, we would need reliable scalings for the $\mathrm{H}$-mode threshold power and a better understanding of the hysterisis-like behavior of the H-to-L transition. Lacking these, we are making the implicit assumption that our enhanced radiation solutions are above the H-to-L power threshold. Empirical scalings based on the analysis of the H-mode Power Threshold Database for ITER [14] predict a power threshold from a favorable $50 \mathrm{MW}$ to a rather unfavorable 420 MW at full density. Assuming that the $\mathrm{H}$-mode hysterisis effect reduces the power of the $\mathrm{H}$-to- $\mathrm{L}$ transition to half the value of the L-to-H threshold power, it follows that we should allow 25 to $210 \mathrm{MW}$ to cross the separatrix to avoid quenching the $\mathrm{H}$-mode. Note that Table 1 corresponds to the maximum radiation cases and that the radiation can be reduced to allow more power to cross the separatrix by adjusting the concentration of the seeded impurity, as indicated in Figs. 5-8. 


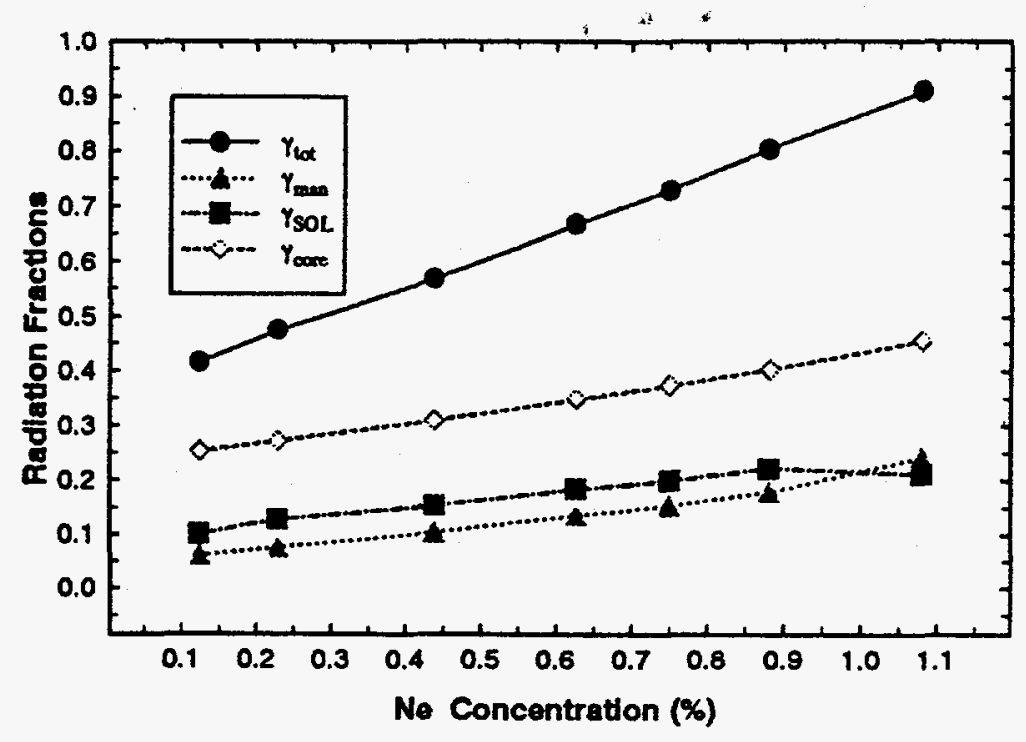

Fig. 5: Radiation fractions from different plasma regions vs. Ne concentration.

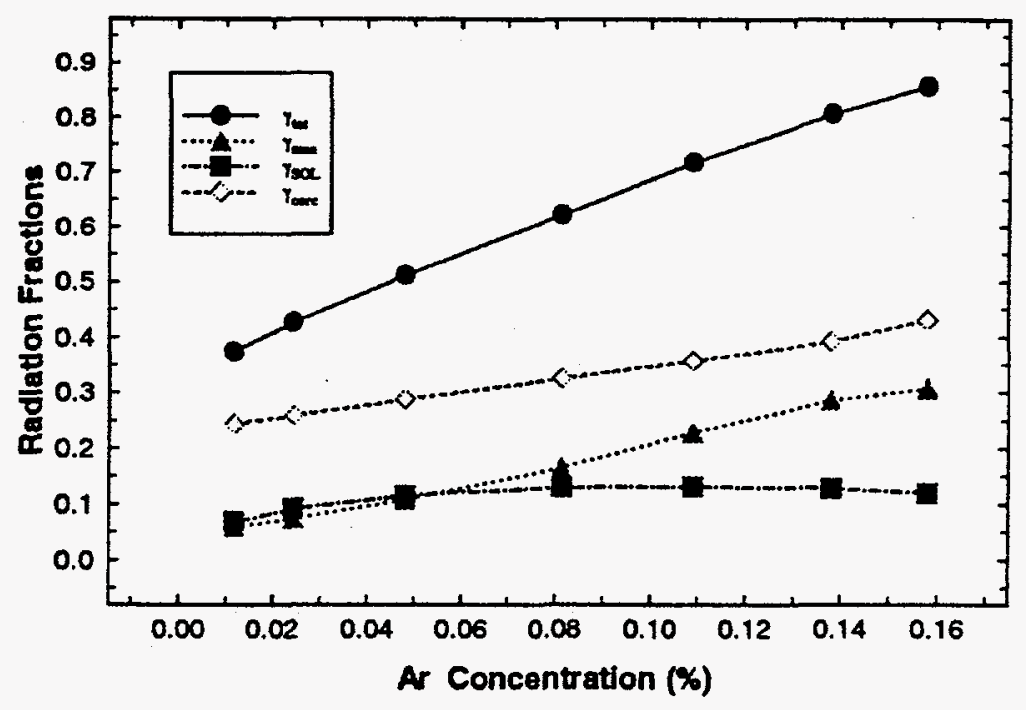

Fig. 6: Radiation fractions from different plasma regions vs. Ar concentration. 


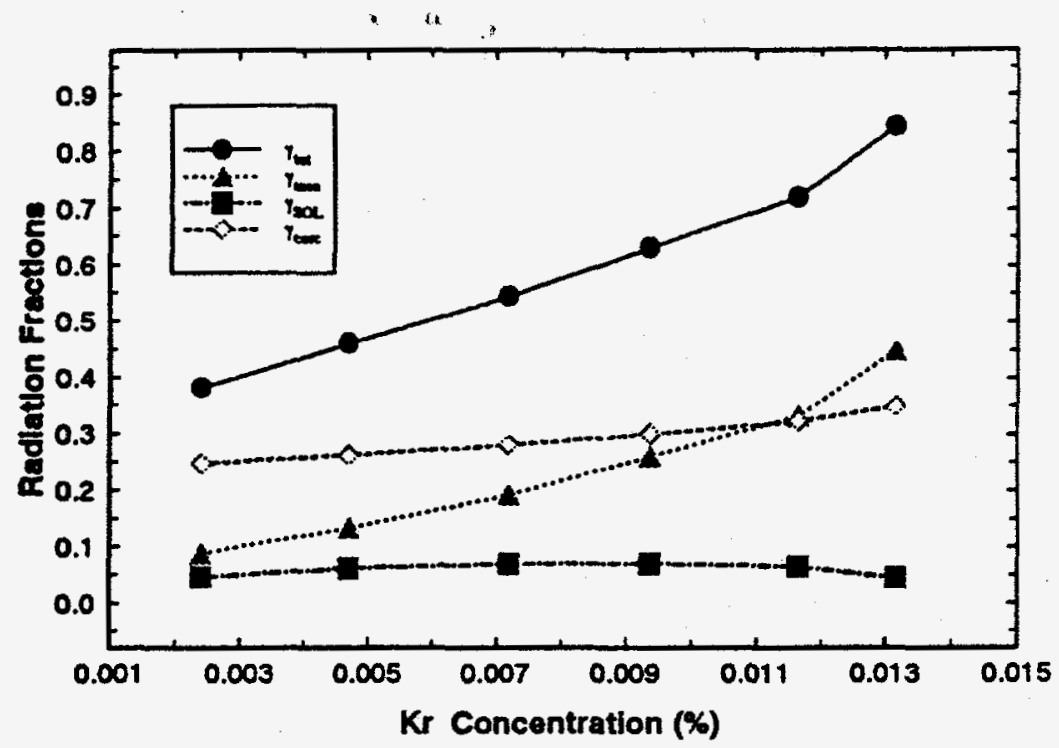

Fig. 7: Radiation fractions from different plasma regions vs. $\mathrm{Kr}$ concentration.

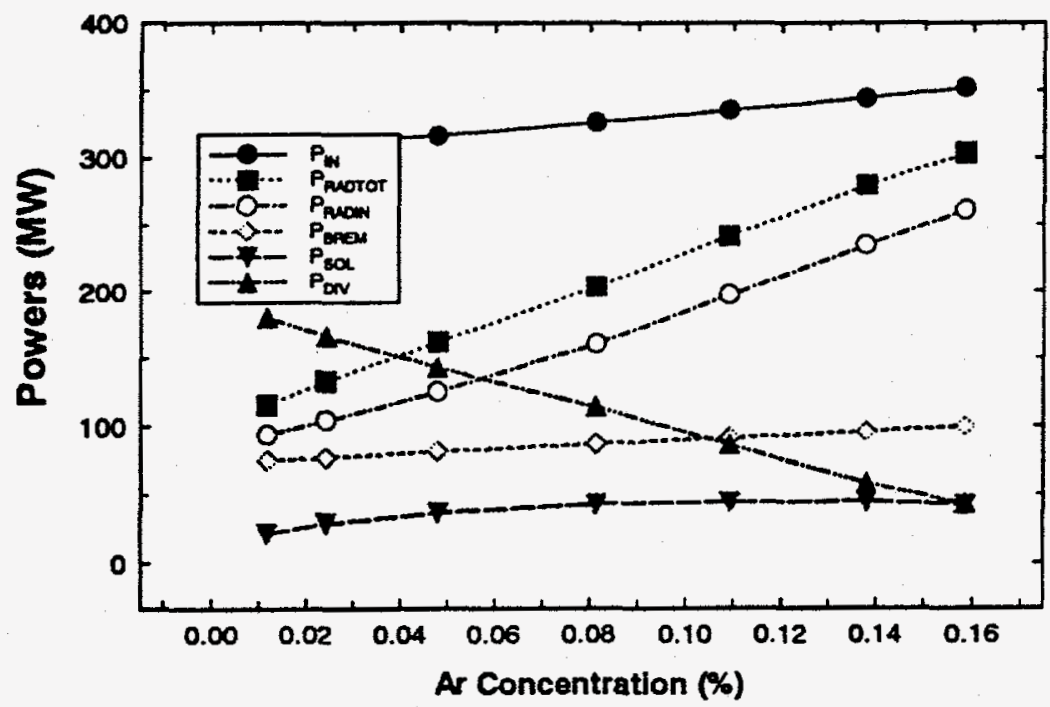

Fig. 8: Global Power quantities vs. Ar concentration.

The radiation power density profiles inside the separatrix are shown in Fig. 9 for $\mathrm{Ar}$ and $\mathrm{Kr}$ for the cases of maximum impurity concentration. For $\mathrm{Ne}$, the power density peaks outside the LCFS, in the SOL/divertor region. It can be seen that the impurity radiation is localized near the plasma edge outside the $q=2$ surface which is located at $\rho$ $=0.83$. In Fig. 10, the integrated power balance profiles for the $\mathrm{Kr}$ maximum concentration case are shown. The reduction of the total convected power crossing each 
flux surface, $P_{\text {con }}$, due to the radiation of the total input power $\left(P_{\text {in }}=P_{\text {fusion }}+P_{\text {aux }}\right)$ can be seen very clearly.

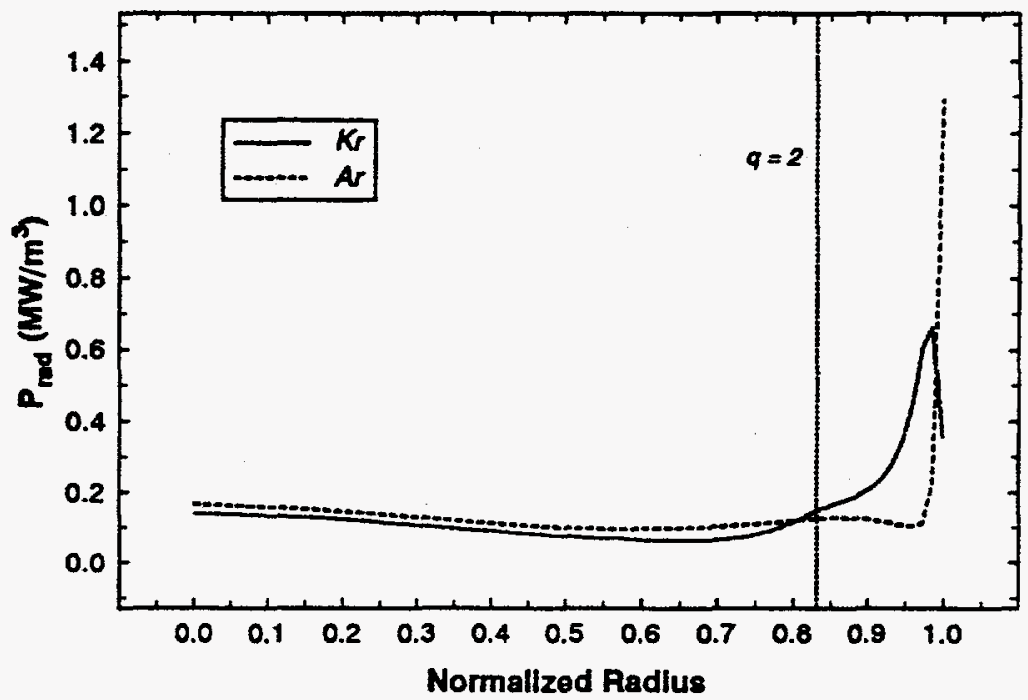

Fig. 9: Radiation power density profiles for $\mathrm{Ar}$ and $\mathrm{Kr}$ injection at maximum concentrations. The location of the $q=2$ surface is also shown. The separatrix is at $\rho=1$.

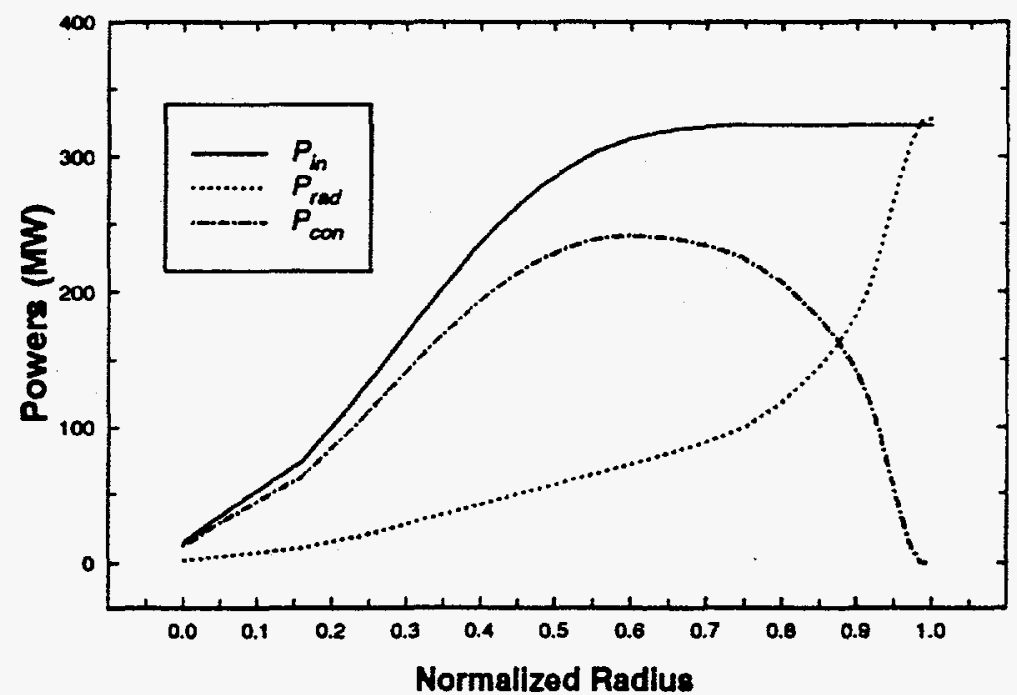

Fig. 10: Integrated power profiles for $\mathrm{Kr}$. The total radiation power $P_{\text {rad, }}$ input power $P_{\text {in }}$ $=P_{\text {fusion }}+P_{\text {aux }}$, and convected plus conducted power crossing each flux surface $P_{c o n}$, are shown. 


\subsection{Impurity Pinch Sensitivity Analysis}

An important concern when considering the introduction of extrinsic impurities into the edge of a tokamak plasma is that they may eventually accumulate in the plasma core quenching the thermonuclear burn. While our simulations presented in the previous section showed that such accumulation does not occur when using our reference transport model, uncertainties in the impurity transport and the fact that such impurity accumulation has been observed in some experiments and is predicted by some transport theories suggest that this is an important issue.

In this section, we present results from a series of simulations in which an inward pinch term was added to the diffusion of the impurity charge states. The pinch term has the form,

$$
v_{z}^{\text {pinch }}=-2 C_{v z} D_{z} \frac{\rho}{a^{2}}
$$

where $C_{V Z}$ is an arbitrary multiplier and $D_{Z}$ is the impurity diffusion coefficient, which is assumed to be equal to the main ion diffusion coefficient. No pinch term is applied to the transport of the main ions.

To assess the sensitivity of our simulations to the uncertainties in the magnitude of this inward pinch term, the multiplier $C_{V z}$ was varied between 0 and 2.5 , a range encompassing the experimentally inferred values of $C_{V z}[15]$.

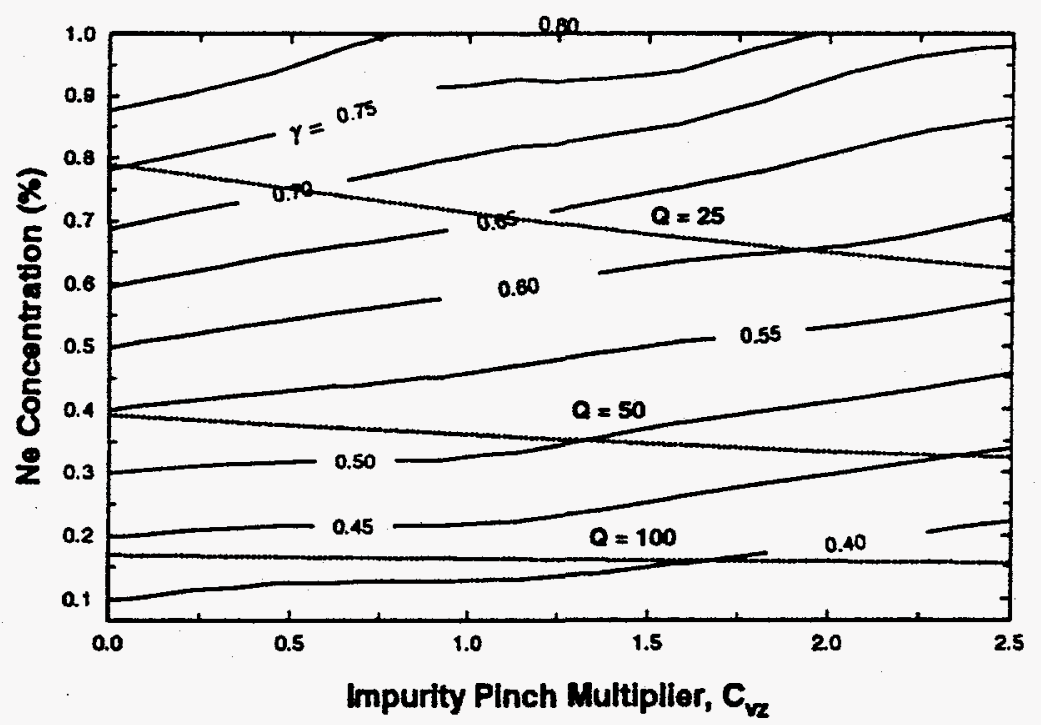

Fig. 11: Contours of constant total radiation fraction $\gamma=P_{r a d}^{\text {tot }} / P_{\text {in }}$ for different values of $C_{V Z}$ and Ne impurity concentration. Contours of constant $Q=P_{\text {fus }} / P_{\text {aux }}$ are also shown.

The results of our simulations are summarized in Figs. 11-15. In Figs. 11-13, contours of constant total radiation fractions $\gamma$ are plotted for a range of impurity pinch multipliers $C_{V Z}$ and volume average impurity concentrations for $\mathrm{Ne}, \mathrm{Ar}$ and $\mathrm{Kr}$. Contours of selected values of the plasma $Q$ are also shown on the same plots. It can be seen that for higher values of the multiplier $C_{V z}$, higher impurity concentrations are required in order 
to achieve the same $\gamma$, while at the same time the plasma performance drops since larger amounts of auxiliary power are required to offset the increased radiation from the plasma core. It is important to notice however, that even at the highest value of $C_{V Z}$ it still possible to radiate $55-65 \%$ of the input power without violating the performance constraints.

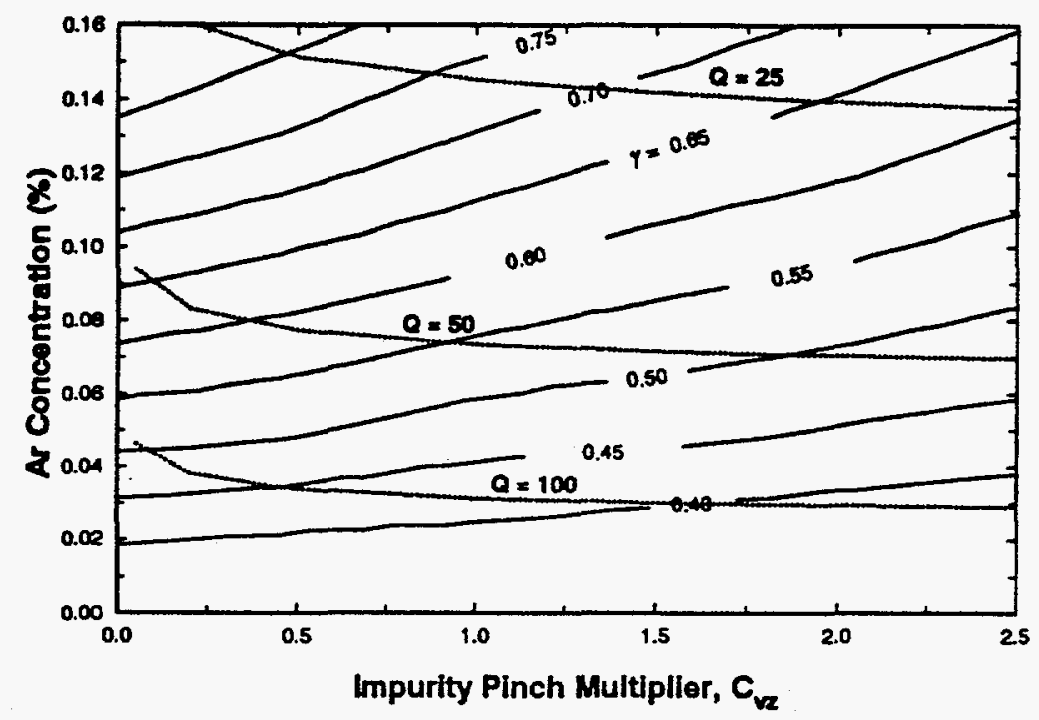

Fig. 12: Contours of constant total radiation fraction $\gamma=P_{\text {rad }}^{\text {tot }} / P_{\text {in }}$ for different values of $C_{V Z}$ and Ar impurity concentration. Contours of constant $Q=P_{\text {fus }} / P_{\text {aux }}$ are also shown.

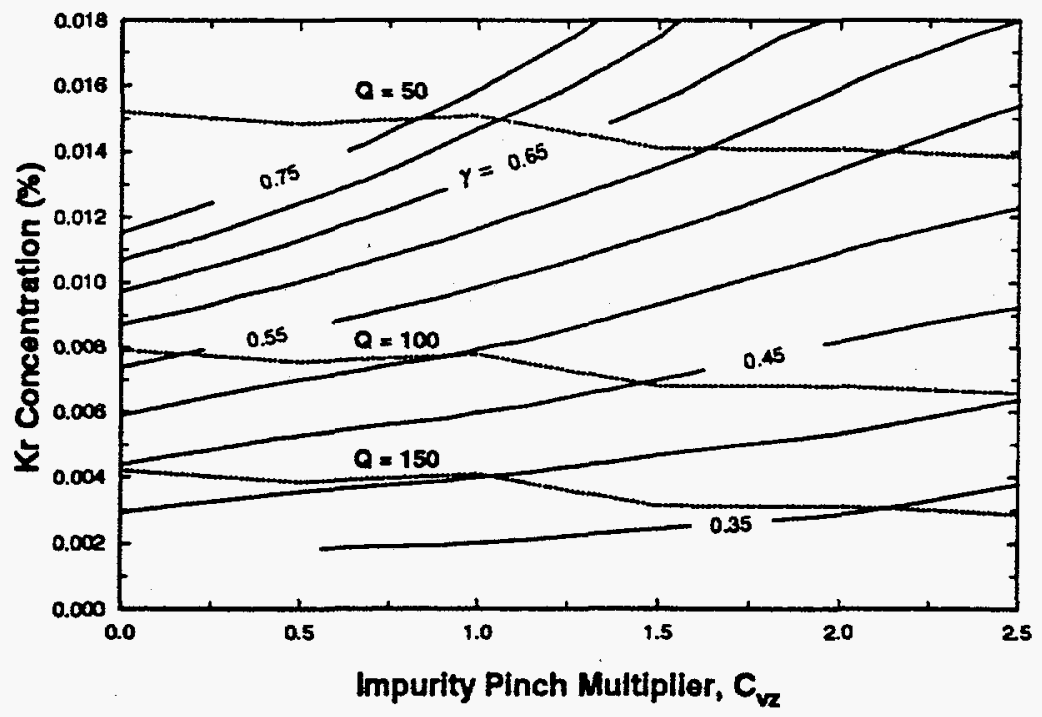

Fig. 13: Contours of constant total radiation fraction $\gamma=P_{\text {rad }}^{\text {rot }} / P_{\text {in }}$ for different values of $C_{V Z}$ and $\mathrm{Kr}$ impurity concentration. Contours of constant $Q=P_{\text {fus }} / P_{\text {eux }}$ are also shown.

In Fig. 14, the mantle fraction, i.e. the ratio of the radiated power from within the plasma mantle (i.e. inside the separatrix but outside the $q=2$ surface) to the total radiated 
power, is plotted vs. the impurity, pinch multiplier $C_{V Z}$ for the maximum allowable concentrations of $\mathrm{Ne}, \mathrm{Ar}$ and $\mathrm{Kr}$. It can be seen that this ratio decreases as $C_{V Z}$ increases, due to the increasing concentration of the radiating impurities in the plasma core.

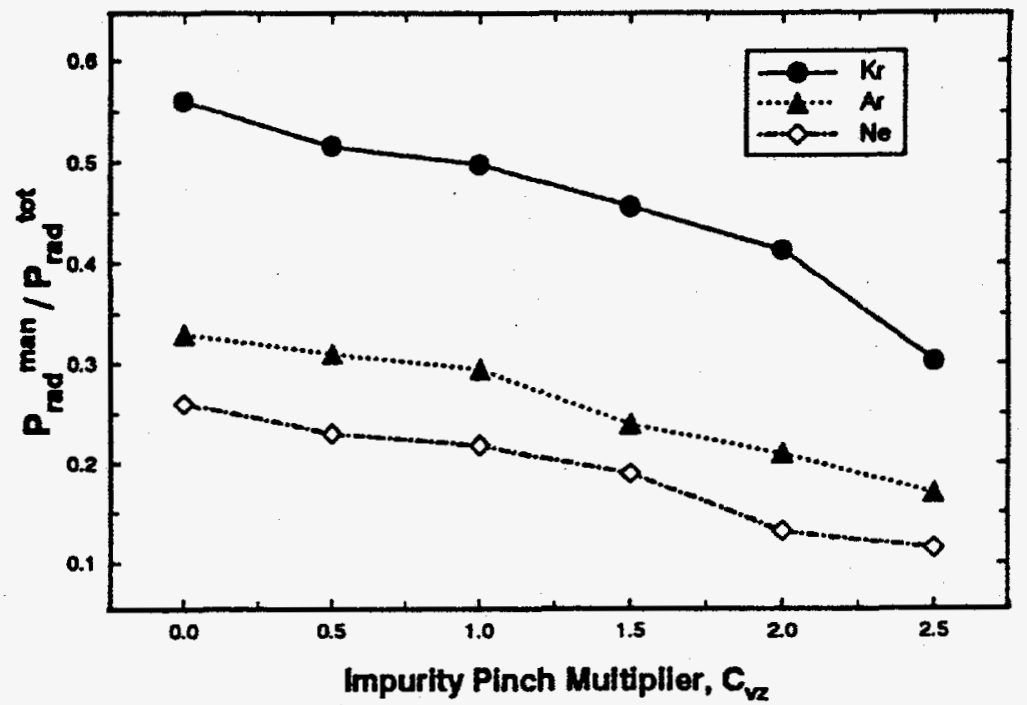

Fig. 14: Fraction of the total radiated power originating from the mantle vs. the impurity pinch multiplier $C_{V z}$ for the maximum concentration $\mathrm{Ne}, \mathrm{Ar}$ and $\mathrm{Kr}$ cases.

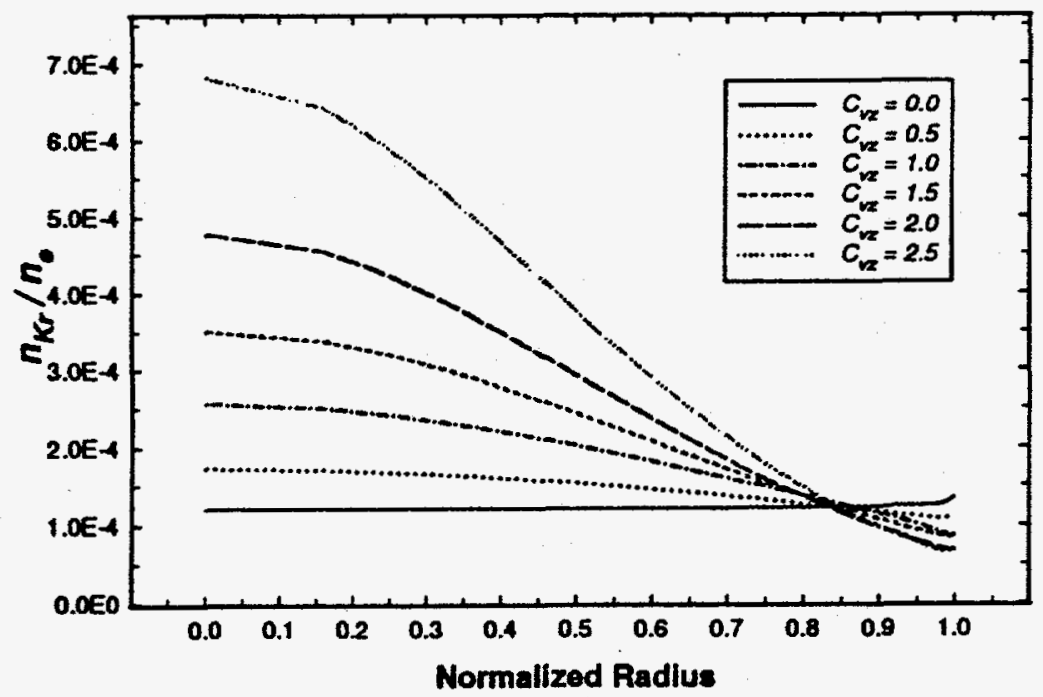

Fig. 15: Profiles of $\mathrm{Kr}$ concentration for different values of the impurity pinch multiplier $C_{V z}$.

Finally, profiles of the impurity concentration for different values of the impurity pinch multiplier $C_{V Z}$ are shown in Fig. 15 for six $\mathrm{Kr}$ cases, each of which has a total radiation fraction $\gamma$ of about $70 \%$. Since the electron density profile is flat, these plots represent also the profiles of the impurity density. 
We conclude from these simulations that a stable, radiative mantle which can exhaust a large fraction of the plasma power can be maintained for a range of possible impurity pinch velocities which includes the range inferred from experiment.

\subsection{Edge Temperature Pedestal Sensitivity Analysis}

The value of the edge temperature pedestal is an important parameter in our calculations, since it determines the lower temperature limit inside the separatrix. Depending on the radiating impurity, higher values of the pedestal may decrease the effective radiating volume (the region where the cooling rate of the injected impurity is near its maximum value) and therefore decrease the amount of power that is radiated from the edge.

Since the exact value of the edge temperature pedestal will depend on the details of the transport processes at the plasma edge, which are largely uncertain, a sensitivity analysis has been performed. The edge temperature pedestal was varied by changing the edge-to-center ratio, $\chi_{e}(1) / \chi_{e}(0)$, in our transport model (Eq. 5). Lower values of this parameter result in higher temperature pedestals. The impurity pinch term has been set to zero for these simulations. Two cases were considered, one with an edge-to-center ratio equal to 0.05 resulting in an edge temperature pedestal of $600 \mathrm{eV}$, and one with a ratio equal to 0.01 resulting in a pedestal of $1500 \mathrm{eV}$. For comparison, the edge temperature pedestal for the reference case was about $340 \mathrm{eV}$. The electron temperature profiles near the plasma edge for the two pedestal cases and the reference case are shown in Fig. 16.

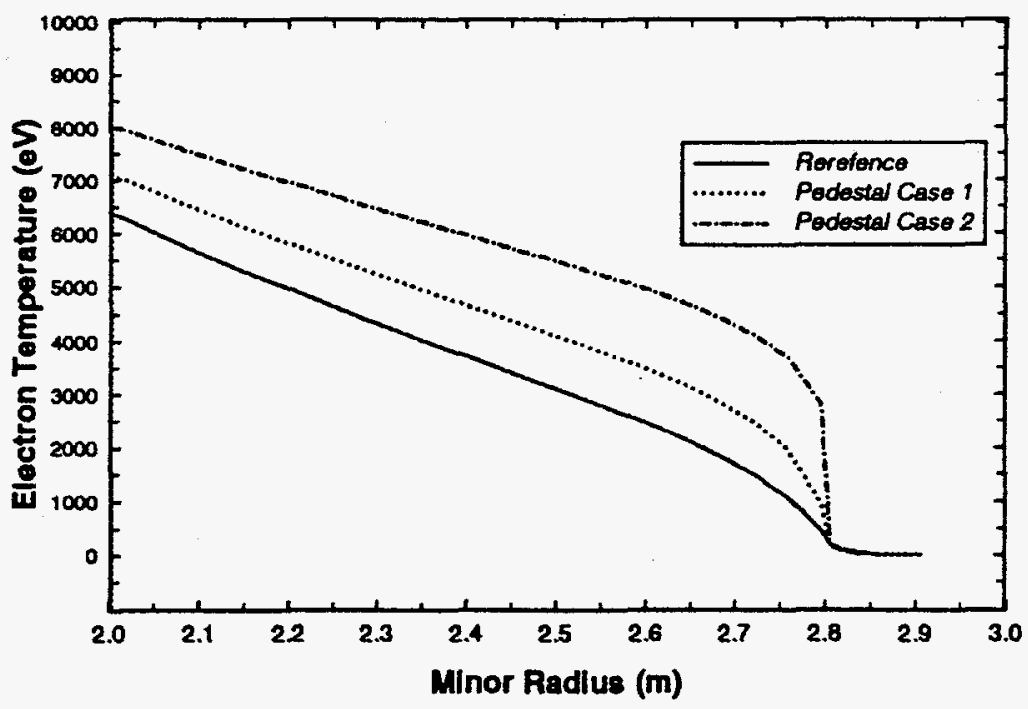

Fig. 16: Electron temperature profiles in the plasma edge region for the reference case and the two pedestal cases.

Once impurity injection begins, the edge temperature drops due to the increased radiation losses in the edge region. This is shown in Fig. 17, where the electron 
temperature at the LCFS is plotted,vs. the total radiated fraction $\gamma$ for the reference case and the two pedestal cases for the case of Ar. Similar results have been obtained for $\mathrm{Ne}$ and $\mathrm{Kr}$.

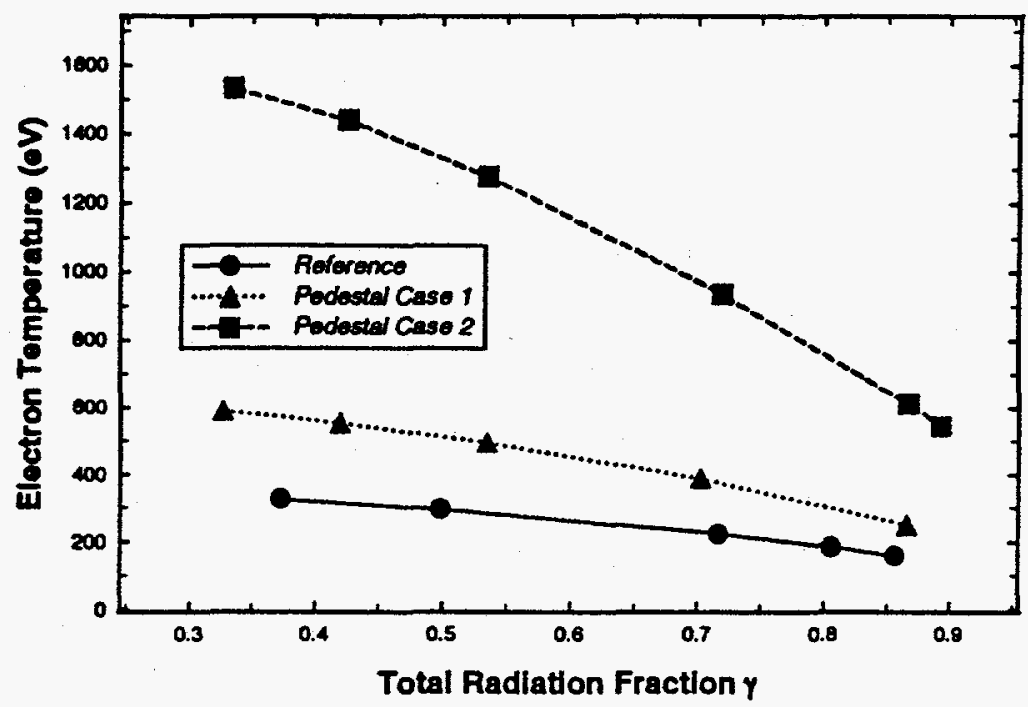

Fig. 17: Electron temperature at the LCFS vs. total radiation fraction during Ar injection.

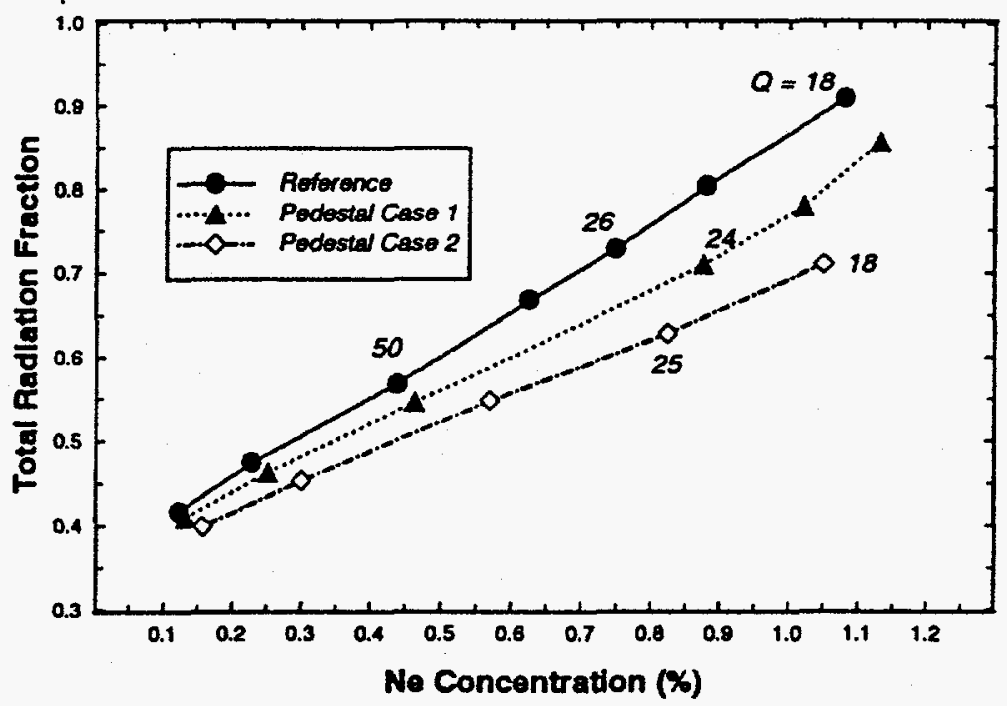

Fig. 18: Total radiation fraction vs. Ne concentration for the reference case and the two pedestal cases. Values of the plasma $Q$ are shown at selected points.

The results of our sensitivity analysis are shown in Figs. 18 and 19. where the total radiation fraction $\gamma$ is plotted vs. the volume average impurity concentration for $\mathrm{Ne}$ and $\mathrm{Ar}$ for the reference case and for the two pedestal cases. Similar results have been obtained for $\mathrm{Kr}$. It can be seen that for higher values of the edge temperature pedestal, higher impurity concentrations are required in order to radiate equivalent amounts of power. The 
explanation for this effect is apparent by looking at Figs. 20-21 where the effective cooling rates, $L_{z}^{\text {sf }}=P_{\text {rad }} /\left(n_{e} n_{z}\right)$, for $\mathrm{Ar}$ and $\mathrm{Kr}$ are plotted vs. the electron temperature and where the mantle temperature ranges are shown, for the maximum impurity concentration cases. It can be seen that higher values of the edge temperature pedestal (before injection) reduce the width of the mantle temperature range and shift it to higher temperatures where the effective cooling rate is lower.

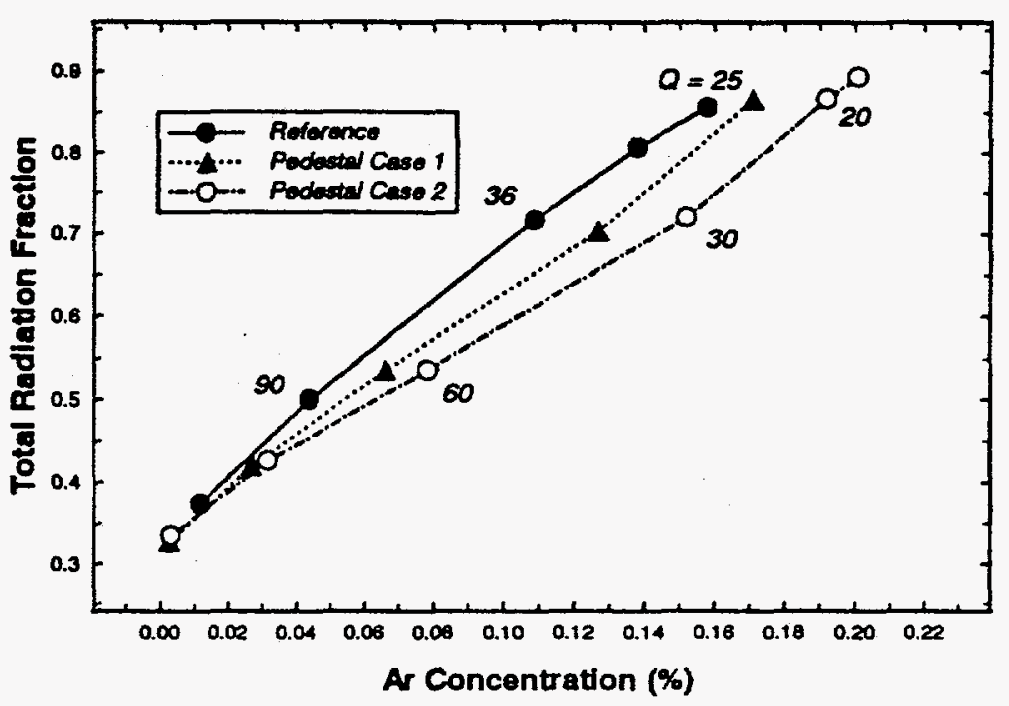

Fig. 19: Total radiation fraction vs. Ar concentration for the reference case and the two pedestal cases. Values of the plasma $Q$ are shown at selected points.

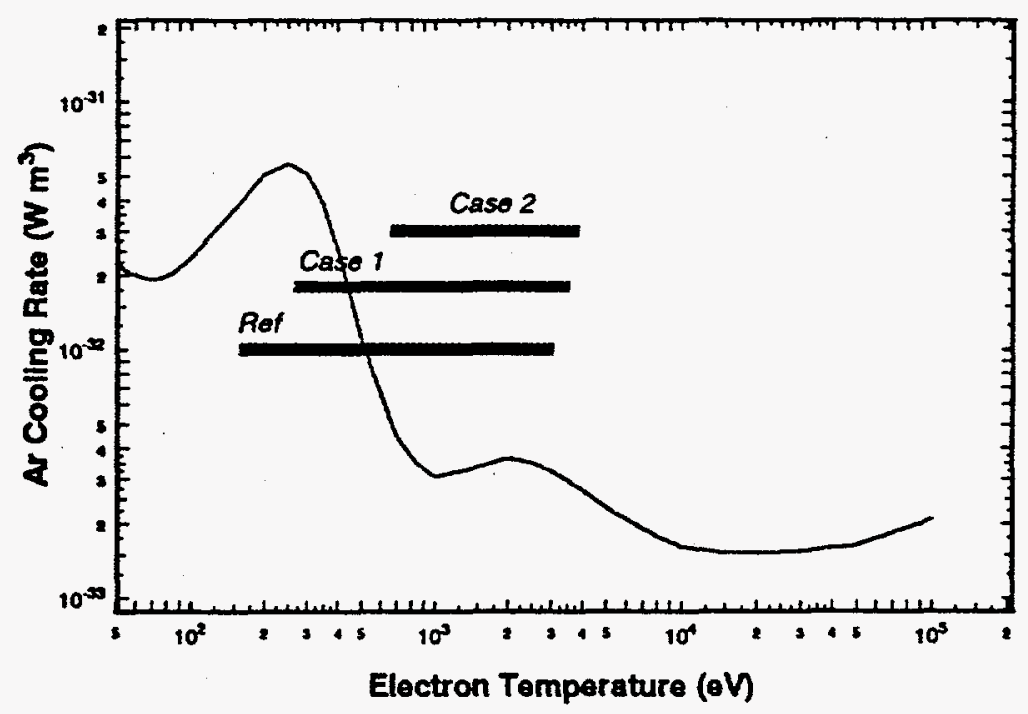

Fig. 20: Ar cooling rate vs. electron temperature. The temperature ranges in the mantle for the reference and the two pedestal cases at maximum impurity concentration are also shown. 


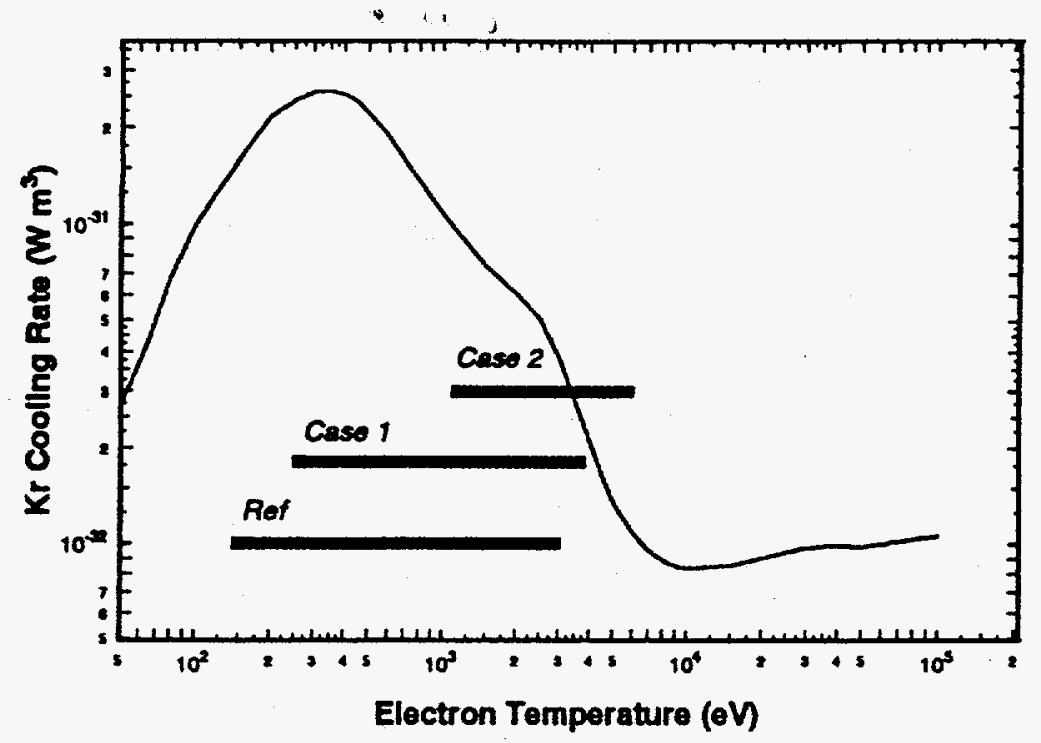

Fig. 21: $\mathrm{Kr}$ cooling rate vs. electron temperature. The temperature ranges in the mantle for the reference and the two pedestal cases at maximum impurity concentration are also shown.

We conclude that it is possible to achieve an effective radiating mantle power exhaust solution over a wide range of pre-injection temperature pedestals.

\subsection{Operation at the Greenwald Density Limit}

The reference volume average electron density for the ITER EDA design is $\left\langle n_{e}\right\rangle=$ $1.3 \times 10^{20} \mathrm{~m}^{-3}$. This value is considerably higher than the Greenwald density limit which is based on operational experience with gas puffing fueling, primarily in ohmic-heated discharges. For ITER the Greenwald limit is

$$
\left(n_{20}\right)_{G}=\frac{I_{M A}}{\pi a^{2}}=0.85
$$

While the density limit issue for ITER has not been resolved yet, we have considered a case with $\left\langle n_{e}\right\rangle \sim 0.85 \times 10^{20} \mathrm{~m}^{-3}$ in order to determine whether radiative edge power exhaust solutions are possible under such low density conditions.

In trying to establish an operating point at the Greenwald limit consistent with the JCT transport guidelines, we found that the constraint on the thermal alpha confinement time, $\tau_{H e}^{*} / \tau_{E}=10$, would result in an He fraction of $14 \%$ and produce low $Q(\leq 21)$ conditions. Since the confinement ratio was initially chosen to yield a $10 \% \mathrm{He}$ concentration at the reference operating point, we choose a value $\tau_{H_{F}}^{*} / \tau_{E}=7$ which results in a $\mathrm{He}$ fraction of 0.1 , in order for our impurity seeding simulations at the Greenwald limit to be comparable to the near-ignited operating points analyzed earlier. In addition, a small inward particle pinch term was added to the diffusion of the main ions, $v_{p}=-2 c_{V} D \rho / a^{2}$, with $c_{V}=0.23$, to help achieve ignition conditions. The resulting 
density profile has a peak-to-average density ratio of 1.12 , slightly more peaked than the flat reference density profile (Fig. 22). No pinch is assumed for the transport of the impurity charge states. The rest of the "Greenwald limit" ignited operating point parameters without impurity injection are: $P_{\text {fusion }}=1.5 \mathrm{GW},\left\langle n_{e}\right\rangle=0.834 \times 10^{20} \mathrm{~m}^{-3},\left\langle T_{e}\right\rangle=$ $17 \mathrm{keV},\left\langle T_{i}>=15.4 \mathrm{keV}\right.$, total radiated power $P_{\mathrm{rad}}=65 \mathrm{MW}$, and total power to the divertor plates $P_{\text {div }}=200 \mathrm{MW}$.

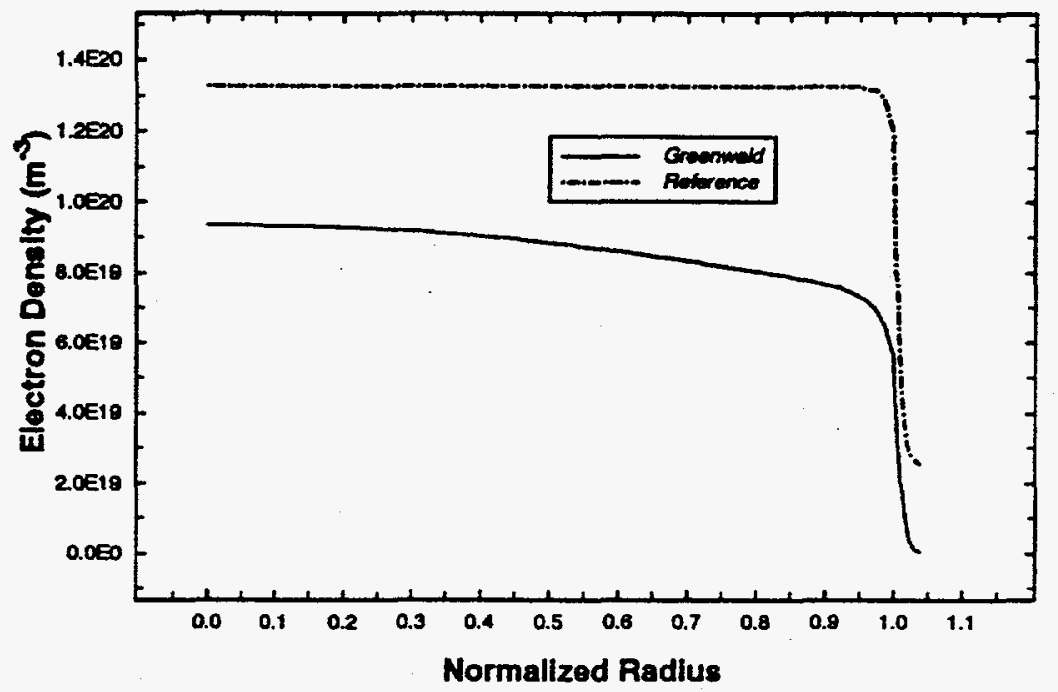

Fig. 22: Electron density profiles for the "Greenwald limit" and reference cases, without impurity injection.

We next considered the injection of various levels of $\mathrm{Kr}$ impurity to reduce the power to the divertor plate in the "Greenwald limit" case. The results of our simulations for $\mathrm{Kr}$ injection are shown in Fig. 23, where the various radiation fractions are plotted versus the volume average $\mathrm{Kr}$ concentration. It can be seen that a substantial fraction of the input power, up to $75 \%$, can be radiated before the plasma $Q$ drops below 25 or the fusion power is reduced more than $5 \%$ from its reference value. At $\gamma=0.75$, about half of the radiated power is from within the plasma mantle. The maximum fraction of power that can be radiated before the plasma edge collapses is $96 \%$.

To compare these results to those for the reference case, the average $\mathrm{Kr}$ concentration and the plasma $Q$ are plotted versus the total radiation fraction $\gamma \equiv P_{\text {rad }} / P_{\text {in }}$ in Figs. 24-26. It can be seen from Fig. 24 that due to the lower densities in the "Greenwald limit" case, higher $\mathrm{Kr}$ concentrations are required in order to radiate equivalent fractions of the input power. This leads to higher values of the central $Z_{\text {eff }}$. While the central $Z_{\text {eff }}$ for the reference case was below 1.65 for the maximum allowable $\mathrm{Kr}$ concentration, it rises to 2.1 for the maximum allowable $\mathrm{Kr}$ concentration in the "Greenwald limit" case.

Performance also suffers in the "Greenwald limit" case compared to the reference case. As can be seen from Fig. 25, the plasma $Q$ is from two to three times lower in the "Greenwald limit" case due to increased heating requirements to offset the increased plasma cooling in the core due to the higher $\mathrm{Kr}$ concentration. The corresponding 
fractional drop in the fusion power ranges from 1.8 to $5 \%$ in the "Greenwald limit" case, compared to below $1 \%$ for the reference case.

From these simulations, we conclude that a range of impurity-seeded radiative power exhaust solutions exist for a full-power ITER operating point at the Greenwald limit.

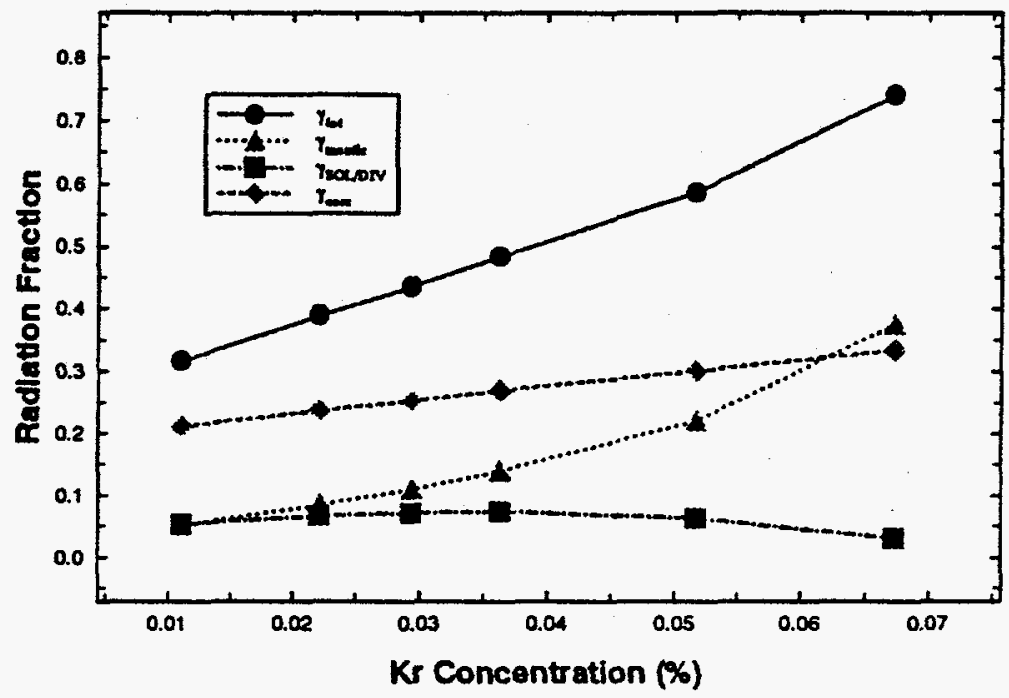

Fig. 23: Radiation fractions from different plasma regions vs. $\mathrm{Kr}$ concentration for the "Greenwald limit" case.

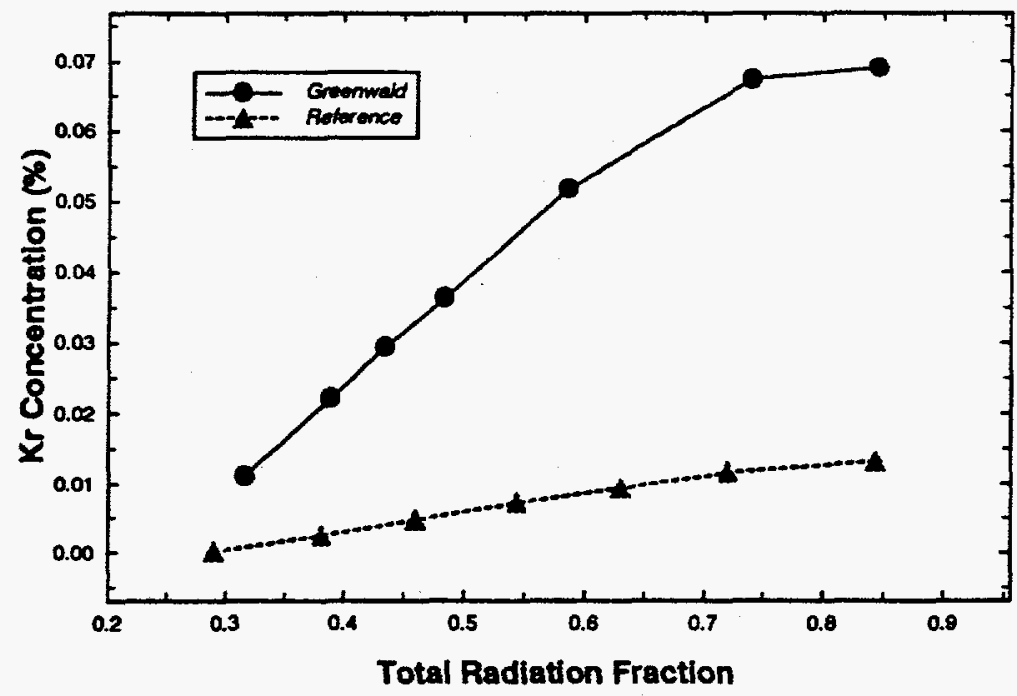

Fig. 24: Average $\mathrm{Kr}$ concentration vs. total radiation fraction $\gamma$, for the "Greenwald limit" and reference cases. 


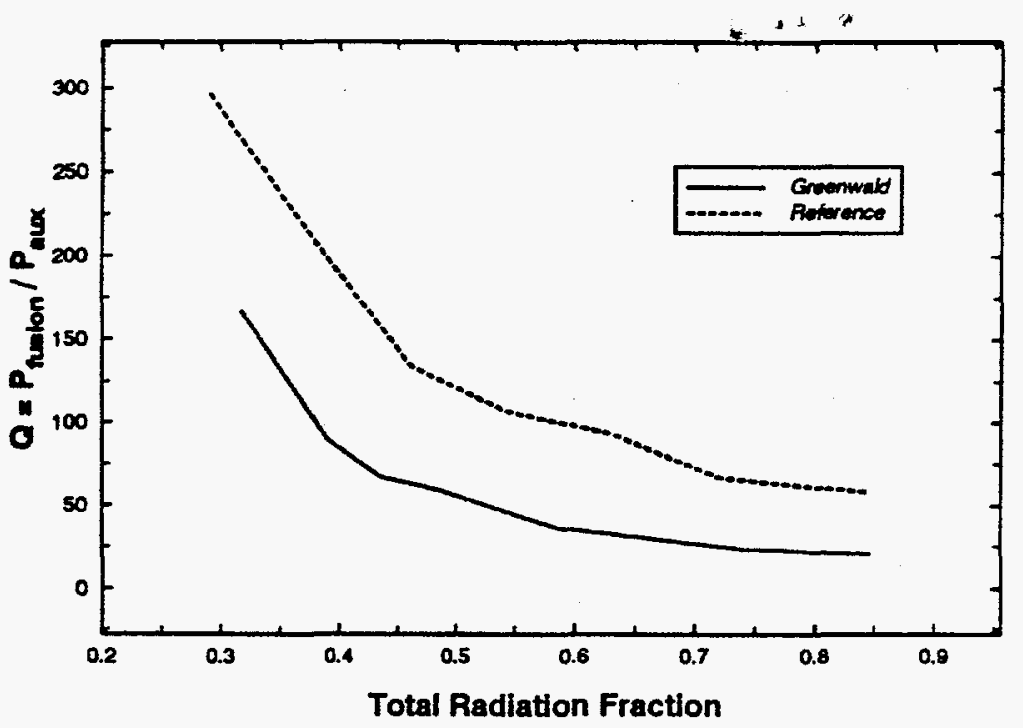

Fig. 25: Plasma $Q=P_{\text {fusion }} / P_{\text {aux }}$ vs. total radiation fraction $\gamma$ for the "Greenwald limit" and reference cases.

\subsection{SUMMARY AND CONCLUSIONS}

We have performed a series of $11 / 2-D$ transport simulations of impurity-seeded radiative power exhaust solutions for ITER. The effect of the seeded impurity on the core power balance was compensated by auxiliary heating. Acceptable solutions were constrained to satisfy $Q>25$ and $\Delta P_{\alpha} / P_{\alpha} \leq 5 \%$. Our emphasis has been on solutions for which a large fraction of the radiating power originates within a "mantle" at the plasma edge - defined herein as the region bound by the $q=2$ surface and the separatrix although we also take into account radiation from the plasma core (inside the $q=2$ surface) and from the scrape-off layer and divertor regions.

We find that up to $70-90 \%$ of the input power can be radiatively exhausted, with almost all of the radiation originating from inside the separatrix, without significant detriment to the plasma power balance, by injecting impurities ranging from $\mathrm{Ne}$ to $\mathrm{Kr}$. The higher- $Z$ impurities are more effective both for producing a large radiating power fraction and for localizing the radiative power exhaust in the mantle where its effect on the core power balance is minimized. The actual radiating fraction from inside the separatrix must be adjusted so that enough power crosses the separatrix to maintain $\mathrm{H}$-mode confinement.

The robustness of the radiative mantle power exhaust solutions to uncertainties in impurity and thermal transport coefficients was investigated. Impurity pinch velocity multipliers in the range $0 \leq C_{V Z} \leq 2.5$, which encompasses the range inferred from experiments, were considered; even at $C_{V z}=2.5$ it was possible to find solutions which radiated 55-65\% of the input power, almost all of it from inside the separatrix, without significant detriment to the core power balance. Radiative mantle solutions capable of exhausting the same fraction of input power were found for different values of the edge 
temperature pedestal (which reşulțed from different $\chi$ profiles), but higher seeded impurity concentrations and lower values of $Q$ were associated with the larger values of the edge temperature pedestals.

A possible ITER ignited operating point at the Greenwald density limit was identified. A range of krypton-seeded solutions capable of radiatively exhausting up to 75 $\%$ of the input power were found.

\section{ACKNOWLEDGMENTS}

This work was supported by the U.S. Department of Energy under contract No. DE-FG05-87ER54122 with the Georgia Tech Research Institute.

\section{REFERENCES}

1. D.E. Post, B. Braams, J. Mandrekas, W. Stacey and N. Putvinskaya, "Power Balance in the ITER Plasma and Divertor," Contrib. Plasma Phys. 36 (1996) 240.

2. J. Mandrekas, W.M. Stacey, "An Impurity Seeded Radiative Mantle for ITER," Nucl. Fusion 35 (1995) 843.

3. J. Mandrekas, W.M. Stacey, F. Kelly, "Impurity Seeded Radiative Power Exhaust Solutions for ITER," Nucl. Fusion 36 (1996) 917.

4. J. Mandrekas, W.M. Stacey, F. Kelly “Radiative Power Exhaust from ITER," Contrib. Plasma Phys. 36 (1996) 245.

5. A.M. Messiaen, J. Ongena, U. Samm, et. al., "Transport and Improved Confinement in High Power Edge Radiation Cooling Experiments on TEXTOR," Nucl. Fusion 36 (1996) 39.

6. G. Telesca, U. Samm, B. Unterberg, et al., "Radiated Power and Ionic Effective Charge During Neon Injection Experiments on TEXTOR," Nucl. Fusion 36 (1995) 347.

7. J. Ongena, A.M. Messiaen, U. Samm, et al., "Confinement Transitions with Radiation Cooling in TEXTOR-94," Plasma Phys. Control. Fusion 38 (1996) 279.

8. J. Neuhauser, M. Alexander, G. Becker, et al., "The Compatibility of High Confinement Times and Complete Divertor Detachment in ASDEX-Upgrade," Plasma Phys. Control. Fusion 37 (1995) A37.

9. R.D. Wood, S.L. Allen, M.E. Fenstermacher, et al., "Neon Impurity Profiles During Radiative Divertor Experiments," Bull. Am. Phys. Soc. 40 (1995) 1832.

10. W. A. Houlberg, S.E. Attenberger, and L.M. Hively, "Contour Analysis of Fusion Reactor Plasma Performance,” Nucl. Fusion 22 (1982) 925.

11. D. Boucher, "Note on Transport Assumptions for ITER Predictions," April 8, 1995

12. D. Boucher, personal communication, February 1996. 
13. K Thomsen, et al., "ITER H Mode Confinement Ḍaţ̧̧base Update," Nucl. Fusion 34 (1994) 131.

14. F. Ryter, et al., "H-Mode Power Threshold Database for ITER," Joint European Torus Report, JET-P (95) 55, November 1995, to be published in Nucl. Fusion.

15. Haddad, C. Belarger, B.C. Gregory, and G. Abel, Plasma Phys. Contr. Fusion, 34, 579 (1992). 